\title{
The Interrelationship between Overweight, Obesity and Hypogonadism among Men
}

\author{
Labban L* \\ Department of Clinical Nutrition, Syria \\ *Corresponding author: Labban L, FADA, CCN, CNC, RD, Professor of Clinical Nutrition, P. O. Box 30440, Damascus, Syria

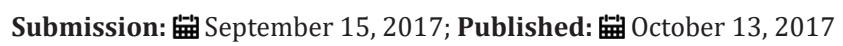

Abbreviations: SHBG: Sex Hormone Binding Globulin

\section{Introduction}

Overweight and Obesity are conditions which are considered as major health risks all over the world. Obese people suffer from at least one disease, or from two or even more chronic diseases associated with obesity, such as type 2 diabetes, hypertension, cardiovascular disease, gallbladder disease, and cancers [1]. Ageing process in men causes a gradual decline in serum testosterone levels and it's connected to accumulation of the abdominal fats [2]. In men, obesity is the single most important factor associated with low testosterone, overriding the effects of age and co-morbidities. This decline in total testosterone levels is due to the obesityassociated lowering in sex hormone binding globulin (SHBG) [3].

Studies have shown that fat-derived adipokines and proinflammatory mediators may play a role in this central gonadal axis suppression. In addition, preclinical evidence has demonstrated that testosterone deficiency promotes adipose tissue accumulation. This bidirectional relationship between low testosterone levels and obesity is supported by clinical studies. Weight loss increases testosterone proportionally to weight loss and testosterone treatment reduces body fat making this hormone a potential treatment of overweight and obesity [4].

On the other hand, obesity is associated with hypogonadism. While this association is widely accepted, the underlying mechanisms remain unclear. Furthermore, obesity is a risk factor for hypogonadism and equally hypogonadism may be a risk factor for obesity. Morbidly obese men that underwent a Rouxen-Y gastric bypass operation noticed an improvement in their testosterone level with improvements in hypogonadal symptoms, which allowed discontinuation of exogenous testosterone therapy. This demonstrates reversal of hypogonadism following weight loss with restoration of gonadal function [5].
There is a significant evidence of testosterone importance in the development and treatment of obesity. Interventional studies offer potential answers to this relationship. These studies have shown that restoring testosterone levels have favorable effects on visceral adiposity and may improve insulin sensitivity and lipid profiles. It is no exaggeration to say that in modern medicine and in the science of endocrinology, testosterone is no longer a marginal hormone and can be used in prevention and treatment of obesity and its related health complications [6]. The fear that testosterone administration to elderly men may increase the risk of prostate cancer is not justified. It only requires prudence in clinical management and should only be recommended by health care provider with an extensive experience.

\section{References}

1. Must A, McKeown NM (2012) The Disease Burden Associated with Overweight and Obesity. In: De Groot LJ, Chrousos G, Dungan K, et al. (Eds.) Endotext [Internet]. South Dartmouth (MA): MDText.com, Inc. 2000 .

2. Stanworth, RD, Jones TH (2008) Testosterone for the aging male; current evidence and recommended practice. Clin Interv Aging 3(1): 25-44.

3. Fui MN, Dupuis P, Grossmann M (2014) Lowered testosterone in male obesity: mechanisms, morbidity and management. Asian Journal of Andrology 16(2): 223-231.

4. Muraleedharan V, Jones TH (2010) Testosterone and the metabolic syndrome. Ther Adv Endocrinol Metab 1(5): 207-223.

5. Zouras S, Stephens JW, Price D (2017) Obesity-related hypogonadism: a reversible condition. BMJ Case Rep. 2017 pii: bcr-2017-220416.

6. Saad F, Gooren LJ (2011) The Role of Testosterone in the Etiology and Treatment of Obesity, the Metabolic Syndrome, and Diabetes Mellitus Type 2. J Obes pii: 471584. 